\title{
Quantum information metric of conical defect
}

\author{
Chong-Bin Chen, ${ }^{1,2, *}$ Wen-Cong Gan, ${ }^{1,2,}$ Fu-Wen Shu, ${ }^{1,2, *}$ and Bo Xiong ${ }^{1, \S}$ \\ ${ }^{1}$ Department of Physics, Nanchang University, Nanchang 330031, China \\ ${ }^{2}$ Center for Relativistic Astrophysics and High Energy Physics, Nanchang University, \\ Nanchang 330031, China
}

(Received 17 May 2018; published 10 August 2018)

\begin{abstract}
A concept of measuring the quantum distance between two different quantum states that is called quantum information metric has been presented. The holographic principle (anti-de Sitter/conformal field theory) suggests that the quantum information metric $G_{\lambda \lambda}$ between perturbed state and unperturbed state in field theory has a dual description in the classical gravity. In this work we calculate the quantum information metric of a theory that is dual to a conical defect geometry and we show that it is $n$ times the one of its covering space. We also give a holographic check for our result in the gravity side. Meanwhile, it was argued that $G_{\lambda \lambda}$ is dual to a codimension-one surface in spacetime and satisfies $G_{\lambda \lambda}=n_{d} \cdot \operatorname{Vol}\left(\Sigma_{\max }\right) / L^{d}$. We show that the coefficient $n_{d}$ for conical defect should be rescaled by $n^{2}$ from the one for anti-de Sitter space. A limit case of conical defect—the massless Banados-Teitelboim-Zannelli (BTZ) black hole-is also considered. We show that the quantum information metric of a massless BTZ black hole disagrees with the one obtained by taking the vanishing temperature limit in BTZ black hole. This provides a new arena in differentiating the different phases between BTZ spacetime and its massless cousin.
\end{abstract}

DOI: 10.1103/PhysRevD.98.046008

\section{INTRODUCTION}

In the last two decades, our understanding of quantum gravity has been greatly enriched because of the advent of the anti-de Sitter/conformal field theory (AdS/CFT) correspondence conjecture [1,2]. This conjecture has been expanded to gauge/gravity correspondence, a duality between more general spacetime background and quantum field theory. A holographic dictionary was established so that one can understand the physical quantities in gravity from its dual field theory equivalently, and vice versa. One of the most famous dictionaries of the past decade is the Ryu-Takayanagi (RT) formula [3,4]. By computing the area of a codimension-two extremal surface in the bulk, one can obtain the entanglement entropy in the quantum field on the boundary that is usually difficult to calculate. Moreover, people begin to realize that quantum information theory plays a crucial role in our understanding of gravity. More information theory quantities, like conditional entropy and

\footnotetext{
*cchongb23@gmail.com

'ganwencong@gmail.com

*shufuwen@ncu.edu.cn

\$stevenxiongbo@gmail.com
}

Published by the American Physical Society under the terms of the Creative Commons Attribution 4.0 International license. Further distribution of this work must maintain attribution to the author(s) and the published article's title, journal citation, and DOI. Funded by SCOAP ${ }^{3}$. mutual information, have been introduced in discussion of gravitational issues.

One recent example is the proposal that the volume of an Einstein-Rosen bridge in spacetime is dual to the computational complexity of the corresponding quantum states of conformal field theory [5-7]. This proposal drove a number of investigations that make us consider the dual theory of the codimension-one volume. Moreover, a quantum information quantity called the quantum information metric (QIM), which measures the distance between two infinitesimally different states,

$$
|\langle\Psi(\lambda+\delta \lambda) \mid \Psi(\lambda)\rangle|=1-G_{\lambda \lambda} \delta \lambda^{2}+\mathcal{O}\left(\delta \lambda^{3}\right),
$$

was taken into account in the dictionary. $|\Psi(\lambda)\rangle$ is a oneparameter family state and the QIM $G_{\lambda \lambda}$ is defined as minus the coefficient of the second order term of the absolute value of this overlap. In [8,9] the QIM was argued to be dual to the volume of a maximal spacelike surface

$$
G_{\lambda \lambda}=n_{d} \cdot \frac{\operatorname{Vol}\left(\Sigma_{\max }\right)}{L^{d}}
$$

where $n_{d}$ is a coefficient, $d$ is the spacetime dimension, $\Sigma$ is a spacelike surface, and $L$ is the radius of the $\operatorname{AdS}_{d+1}$ spacetime. This new dictionary may shed light on our understanding of codimension-one surface in holography (see e.g., [10]). 
In this paper we consider the QIM of a dual CFT for conical defect geometry in three dimensions. In bulk side, conical defect spacetime is a solution of the Einstein equations with static particle source at the origin [11,12]. It can be constructed by angular identification $\theta \sim \theta+2 \pi / n$ on a pure $\mathrm{AdS}_{3}$ spacetime. The conical defect can be regarded as a particular excited state so it is different from the vacuum AdS space. In pure AdS space, it is possible to use the RT surfaces to probe all of the spacetime region if we consider the largest one, which is associated to the entanglement of half of the CFT on the boundary. However, when we consider the conical defect that is away from pure AdS space, a so-called entanglement shadow that cannot be probed by minimal surfaces appears $[13,14]$. To recover the physics in the entanglement shadows one should take into account the "internal" degrees of freedom (d.o.f.) of the dual CFT that is not spatially organized. In this way we give a computation of the QIM for such conical defect dual CFT through the correlation function in the field theory. We find that the QIM of a conical defect is $n$ times the one of its covering space, which is pure $\mathrm{AdS}_{3}$. Our calculation suggests that this result is related to the fact that the central charge of a conical defect is increased by a factor of $n: c=n \tilde{c}$. To verify our result, we also perform a holographic computation in the bulk. To proceed, we notice that the operator in CFT is dual to a local field in the bulk: $\Phi(z, x) \leftrightarrow \mathcal{O}(x)$. And the holographic relation $Z_{\mathrm{CFT}}=Z_{\text {bulk }}$ implies that one can turn to the computation of the information metric in the gravity side [15]. Our results suggest that when we add a scalar field perturbation to the spacetime background, all scalar fields in copy of images should be considered. It gives the same result as the CFT computation. We also compare our result with (2) in the conical defect and its covering space. We find that for the conical defect this proposal also holds, but the coefficient $n_{d}^{\text {defect }}$ is rescaled by a factor $n^{2}$, i.e., $n_{2}^{\text {defect }}=n^{2} \cdot n_{2}^{\text {AdS }}$. We argue that this factor comes from the $\mathbb{Z}_{n}$ quotient on the original space, which is independent of dimension $d$. We also discuss the case $n \rightarrow \infty$, whose metric is a massless Banados-TeitelboimZannelli (BTZ) black hole. The massless BTZ has vanishing horizon so that one can approach the singularity by infinitely winding geodesics. We find that the QIM of massless BTZ is inconsistent with the result that is obtained by taking limit $\beta \rightarrow \infty$ in the information metric of BTZ.

In Sec. II we review the conical defect geometry and its CFT dual. We get the Virasoro algebra and correlation function of operators $\mathcal{O}$ of a conical defect dual CFT. In Sec. III we present the calculation of QIM by path integral in field theory. We use this formulation to obtain the QIM of the conical defect dual theory. We also recover the result from holographic perspective in the dual gravity side and then compare this result with the proposal (2). In Sec. IV we consider a limit case of the conical defect, which is the massless BTZ black hole case and make some remarks on it. Conclusions are made in the last section.

\section{CONICAL DEFECT GEOMETRY AND ITS CFT DUAL}

The conical defect geometry $\mathrm{AdS}_{3} / \mathbb{Z}_{n}$ can be obtained by an angular identification of a global $\mathrm{AdS}_{3}$ geometry. Its metric is the same as the global $\mathrm{AdS}_{3}$ except the periodicity of the angular coordinate $\theta$ is $2 \pi / n$ rather than $2 \pi$; i.e., $\theta \sim \theta+2 \pi / n$ (in general, $n$ need not be an integer, but the subalgebra of asymptotic symmetries has the Virasoro form only if $n \in \mathbb{Z}_{+}$[16]; we only consider integer $n$ in this paper),

$d s^{2}=-\left(1+\frac{r^{2}}{L^{2}}\right) d t^{2}+\left(1+\frac{r^{2}}{L^{2}}\right)^{-1} d r^{2}+r^{2} d \theta^{2}$.

This is a solution of the negative constant-curvature Einstein equations with a static particle source placed at $r=0[11,12]$. Identifying $\theta \sim \theta+2 \pi / n$ makes the space a cone; then the metric is not well defined at its vertex, where $r=0$. It is useful to change the coordinate from (3) by a rescaling

$$
\theta^{\prime}=n \theta \quad \text { and } \quad r^{\prime}=r / n \quad \text { and } \quad t^{\prime}=n t,
$$

which results in

$$
d s^{2}=-\left(\frac{1}{n^{2}}+\frac{r^{\prime 2}}{L^{2}}\right) d t^{\prime 2}+\left(\frac{1}{n^{2}}+\frac{r^{\prime 2}}{L^{2}}\right)^{-1} d r^{\prime 2}+r^{\prime 2} d \theta^{\prime 2} .
$$

Now the periodicity of the new angular coordinate is $2 \pi$, i.e., $\theta^{\prime} \sim \theta^{\prime}+2 \pi$, but there is still a singularity on $r^{\prime}=0$. From these we can regard the pure $\mathrm{AdS}_{3}$ as the covering space of the conical defect space. We denote (3) as covering coordinates and (5) as defect coordinates. We have to point out that if we consider a cutoff of the infinite boundary $r=1 / \epsilon$, we also need to rescale the cutoff after this change of coordinates $\epsilon^{\prime}=n \epsilon$, where $1 / \epsilon$ and $1 / \epsilon^{\prime}$ are the IR cutoffs in covering coordinates and defect coordinates, respectively.

The field theory dual to a conical defect has been studied for a number of years [16-18]. One approach is regarding the conical defect as an excited state of a pure $\mathrm{AdS}_{3}$ by adding a static particle on the origin [12,18]. In the CFT picture the vacuum state is dual to the vacuum $\mathrm{AdS}_{3}$ and the conical defect is a particular excited state.

According to the Brown-Henneaux method in constructing the asymptotic symmetry algebra of the $\mathrm{AdS}_{3}$, the Virasoro algebra that preserves the diffeomorphisms of the covering space $\mathrm{AdS}_{3}$ is [19]

$$
\left[\tilde{L}_{k}, \tilde{L}_{s}\right]=(k-s) \tilde{L}_{k+s}+\frac{\tilde{c}}{12} k\left(k^{2}-1\right) \delta_{k+s},
$$


where $\tilde{c}$ is the central charge of the covering theory. ${ }^{1}$ But in conical defect theory, not all diffeomorphisms descend to defect theory because of the restriction of $\mathbb{Z}_{n}$ symmetry. The Virasoro algebra of the defect theory is a subalgebra generated by $\tilde{L}_{n k}[13,16]$,

$L_{k}=\frac{1}{n} \tilde{L}_{n k}, \quad k \neq 0 \quad L_{0}=\frac{1}{n}\left(\tilde{L}_{0}-\frac{\tilde{c}}{24}\right)+\frac{n \tilde{c}}{24}$.

The defect theory has the central charge $c=n \tilde{c}$. Since the central charge is related to the $\mathrm{AdS}_{3}$ radius and gravitational constant as $c=3 L / 2 G$, this indeed corresponds to the rescaling of the gravitational constant $\tilde{G}=n G$ in the bulk.

In the CFT, there are some internal d.o.f. that are not spatially organized. The question is how these d.o.f. entangle and how to describe them. The internal d.o.f. are usually gauged. In [13] the authors defined a so-called "entwinement" to probe these gauged d.o.f.. They deal with this problem in this way: first embedding the theory in a larger theory (the covering space) where the d.o.f. are not gauged. After "ungauging" the gauge symmetry we compute the $\mathbb{Z}_{n}$-invariant quantity that is the sum of quantities of all gauged copies in the ungauged theory. For example, we should sum over images of correlation to compute the correlation function of operator $\mathcal{O}$ in dual defect $\mathrm{CFT}_{c}$. The operator $\mathcal{O}$ is given by $[13,20]$

$$
\mathcal{O}(\tau, \theta)=\sum_{k=0}^{n-1} g^{k} \tilde{\mathcal{O}}(\tau, \theta)=\sum_{k=0}^{n-1} e^{i \frac{2 \pi k}{n} \partial \theta} \tilde{\mathcal{O}}(\tau, \theta),
$$

where $g^{k}$ is a $\mathbb{Z}_{n}$ generator. So one can use the operator $\tilde{\mathcal{O}}$ in covering theory $\mathrm{CFT}_{\tilde{c}}$ to compute the two-point function in defect theory $\mathrm{CFT}_{c}$ as

$$
\begin{aligned}
\langle\mathcal{O} & \left.\left(\tau_{1}, \theta_{1}\right) \mathcal{O}\left(\tau_{2}, \theta_{2}\right)\right\rangle \\
& =\sum_{a=0}^{n-1} \sum_{b=0}^{n-1} e^{i \frac{2 \pi a}{n} \frac{\partial}{\partial \theta_{1}}} e^{i \frac{2 \pi b}{n} \frac{\partial}{\partial \theta_{2}}}\left\langle\tilde{\mathcal{O}}\left(\tau_{1}, \theta_{1}\right) \tilde{\mathcal{O}}\left(\tau_{2}, \theta_{2}\right)\right\rangle \\
& =\sum_{a=0}^{n-1} \sum_{b=0}^{n-1}\left\langle\tilde{\mathcal{O}}\left(\tau_{1}, \theta_{1}+\frac{2 \pi a}{n}\right) \tilde{\mathcal{O}}\left(\tau_{2}, \theta_{2}+\frac{2 \pi b}{n}\right)\right\rangle,
\end{aligned}
$$

where we have obtained the expression for the correlation function as a sum over all images.

The correlation function in the conical defect can be correctly constructed from Gubser-Klebanov-PolyakovWitten and geodesic approximation, up to a factor [2022]. There is also a so-called entwinement that describes the internal d.o.f. by these long geodesics as formed in [13].

\footnotetext{
${ }^{1}$ In this paper, all quantities associated with the covering space are marked by a tilde to differentiate them from those belonging to the conical defect.
}

These nonminimal geodesics can probe the entanglement shadow in the spacetimes, which is the region the minimal one cannot reach in the conical defect geometry. The same method of images for computing Green functions was also used in BTZ spacetime [17,23-25]. In the next section we use (9) to compute the correlation function in the dual boundary theory of the conical defect $\left(\mathrm{CFT}_{c}\right)$ and to see the relation with the one in the dual boundary theory of the covering space $\left(\mathrm{CFT}_{\tilde{c}}\right)$.

\section{QUANTUM INFORMATION METRIC FOR CONICAL DEFECT}

\section{A. CFT computation}

In this subsection we try to calculate the QIM of a CFT dual to a conical defect geometry from the boundary theory side. We first clarify the analysis in [8,9] for the general $d$-dimensional CFT and then turn to discuss the conical defect case. More details are in Appendix A.

We consider a $d$-dimensional unperturbed CFT whose Euclidean Lagrangian is $\mathcal{L}_{0}$ and the corresponding ground state is $\left|\Psi_{0}\right\rangle=|\Psi(\tilde{\lambda}=0)\rangle$, where $\tilde{\lambda}$ is the parameter of this state. Now one can add a little deformation to this original CFT by adding $\delta \mathcal{L}_{0}=\delta \tilde{\lambda} \tilde{\mathcal{O}}$ to $\mathcal{L}_{0}$, so the perturbed Lagrangian can be denoted by $\mathcal{L}_{1}=\mathcal{L}_{0}+\delta \mathcal{L}_{0}$, and the corresponding ground state of perturbed field is denoted by $\left|\Psi_{1}\right\rangle=|\Psi(\tilde{\lambda}+\delta \tilde{\lambda})\rangle$. The difference between the unperturbed state and the perturbed one is given by

$$
\left\langle\Psi_{1}(\epsilon) \mid \Psi_{0}\right\rangle=\frac{\left\langle\exp \left(-\int_{\epsilon}^{\infty} d \tau \int d^{d-1} x \delta \tilde{\lambda} \tilde{\mathcal{O}}(\tau, x)\right)\right\rangle}{\left\langle\exp \left(-\left(\int_{-\infty}^{-\epsilon}+\int_{\epsilon}^{\infty}\right) d \tau \int d^{d-1} x \delta \tilde{\lambda} \tilde{\mathcal{O}}(\tau, x)\right)\right\rangle^{1 / 2}}
$$

where $\left|\Psi_{1}(\epsilon)\right\rangle$ is the regularized ground state to which we introduce a cutoff $\epsilon$ at $\tau=0$ because there is a discontinuity at this location; see (A6) in Appendix A. The QIM is defined by minus the coefficient of the second order term of the absolute value of this inner product,

$$
\left|\left\langle\Psi_{1}(\epsilon) \mid \Psi_{0}\right\rangle\right|=1-G_{\tilde{\lambda} \tilde{\lambda}} \delta \tilde{\lambda}^{2}+\tilde{\mathcal{O}}\left(\delta \tilde{\lambda}^{3}\right) .
$$

It is not difficult to obtain its expression from (10),

$$
\begin{aligned}
G_{\tilde{\lambda} \tilde{\lambda}}= & \frac{1}{2} \int d^{d-1} x_{1} \int d^{d-1} x_{2} \int_{-\infty}^{-\epsilon} d \tau_{1} \\
& \times \int_{\epsilon}^{\infty} d \tau_{2}\left\langle\tilde{\mathcal{O}}\left(\tau_{1}, x_{1}\right) \tilde{\mathcal{O}}\left(\tau_{2}, x_{2}\right)\right\rangle,
\end{aligned}
$$

where we have assumed that the one-point function is 0 and have used the reversal symmetry relation of the two-point function. One can also consider the CFT that lives on a cylinder $\mathbb{R} \times S^{d-1}$. The QIM of this case is more nontrivial because it has the universal term in general. The derivation of the QIM of a cylinder is given by [26] 


$$
\begin{aligned}
G_{\tilde{\lambda} \tilde{\lambda}}= & \frac{1}{2} \int d^{d-1} \Omega_{1} \sqrt{g_{S^{d-1}}} \int d^{d-1} \Omega_{2} \sqrt{g_{S^{d-1}}} \int_{-\infty}^{-\epsilon} d \tau_{1} \\
& \times \int_{\epsilon}^{\infty} d \tau_{2}\left\langle\tilde{\mathcal{O}}\left(\tau_{1}, \Omega_{1}\right) \tilde{\mathcal{O}}\left(\tau_{2}, \Omega_{2}\right)\right\rangle,
\end{aligned}
$$

where $g_{S^{d-1}}$ is the determinant of the metric of the sphere and it cannot be ignored for $d>2$. While for $d=2$, it reduces to 1 . In what follows we only consider this case.

Now let us turn to compute the QIM of a field theory that is dual to the conical defect geometry. Before doing that, we should be careful with the normalization constant of the correlation functions. For a CFT that lives on a cylinder, we have $[9,26]$

$$
\left\langle\tilde{\mathcal{O}}\left(\tau_{1}, \theta_{1}\right) \tilde{\mathcal{O}}\left(\tau_{1}, \theta_{2}\right)\right\rangle=\frac{\mathcal{N}_{\Delta, \tilde{\kappa}}}{\left(2 \cosh \left(\tau_{1}-\tau_{2}\right)-2 \cos \left(\theta_{1}-\theta_{2}\right)\right)^{\Delta}}
$$

In order to guarantee consistence with the bulk computation, one chooses the following normalization constant:

$$
\mathcal{N}_{\Delta, \tilde{\kappa}}=\frac{L^{d-1} d \Gamma(\Delta)}{\tilde{\kappa}^{2} \pi^{\frac{d}{2}} \Gamma\left(\Delta-\frac{d}{2}\right)},
$$

which is related to the Newton constant $\mathcal{N}_{\Delta, \tilde{\kappa}} \sim 1 / \tilde{\kappa}^{2}=$ $1 / 8 \pi \tilde{G}$ (one can find the derivation of this constant in Appendix B). It was mentioned in the last section that the gravitational constant for conical defect and covering space is different by a rescaling due to (7). In other words, when we represent quantities of the conical defect in terms of the ones of the covering space, this constant should also be changed. As a consequence, this gives us a rescaling $\tilde{\kappa}^{2}=n \kappa^{2}$.

Now let us see the QIM of the conical defect, by making use of the correlation function of the conical defect (9) and the definition of the QIM (12),

$$
\begin{aligned}
G_{\lambda \lambda}^{\text {defect }} & =\frac{1}{2} \int_{0}^{\frac{2 \pi}{n}} d \theta_{1} \int_{0}^{\frac{2 \pi}{n}} d \theta_{2} \int_{-\infty}^{-\epsilon} d \tau_{1} \int_{\epsilon}^{\infty} d \tau_{2}\left\langle\mathcal{O}\left(\tau_{1}, \theta_{1}\right) \mathcal{O}\left(\tau_{2}, \theta_{2}\right)\right\rangle_{\kappa} \\
& =\frac{1}{2} \sum_{a=0}^{n-1} \sum_{b=0}^{n-1} \int_{0}^{\frac{2 \pi}{n}} d \theta_{1} \int_{0}^{\frac{2 \pi}{n}} d \theta_{2} \int_{-\infty}^{-\epsilon} d \tau_{1} \int_{\epsilon}^{\infty} d \tau_{2}\left\langle\tilde{\mathcal{O}}\left(t_{1}, \theta_{1}+\frac{2 \pi a}{n}\right) \tilde{\mathcal{O}}\left(t_{2}, \theta_{2}+\frac{2 \pi b}{n}\right)\right\rangle_{\kappa}
\end{aligned}
$$

The subscript $\kappa$ means that the normalization constant of the correlation functions is related to Newton constant $\kappa^{2} \sim G$ of the conical defect geometry. Our aim is to find the relation of the QIM between the conical defect $G_{\lambda \lambda}^{\text {defect }}$ and the covering space $G_{\tilde{\lambda} \tilde{\lambda}}^{\mathrm{AdS}}$. Using the identity

$$
\begin{aligned}
\sum_{a=0}^{n-1} \int_{0}^{\frac{2 \pi}{n}} d \theta f\left(\theta+\frac{2 \pi a}{n}\right) & =\sum_{a=0}^{n-1} \int_{\frac{2 \pi a}{n}}^{\frac{2 \pi(a+1)}{n}} d x_{a} f\left(x_{a}\right) \\
& =\int_{0}^{2 \pi} d x f(x),
\end{aligned}
$$

where $x_{a}=\theta+2 \pi a / n$, we can rewrite (16) as

$$
\begin{aligned}
G_{\lambda \lambda}^{\text {defect }} & =\frac{1}{2} \int_{0}^{2 \pi} d x_{1} \int_{0}^{2 \pi} d x_{2} \int_{-\infty}^{-\epsilon} d \tau_{1} \int_{\epsilon}^{\infty} d \tau_{2}\left\langle\tilde{\mathcal{O}}\left(\tau_{1}, x_{1}\right) \tilde{\mathcal{O}}\left(\tau_{2}, x_{2}\right)\right\rangle_{\kappa} \\
& =\frac{n}{2} \int_{0}^{2 \pi} d x_{1} \int_{0}^{2 \pi} d x_{2} \int_{-\infty}^{-\epsilon} d \tau_{1} \int_{\epsilon}^{\infty} d \tau_{2}\left\langle\tilde{\mathcal{O}}\left(\tau_{1}, x_{1}\right) \tilde{\mathcal{O}}\left(\tau_{2}, x_{2}\right)\right\rangle_{\tilde{\kappa}} .
\end{aligned}
$$

In the second line we have changed the Newton constant from $\kappa$ to $\tilde{\kappa}$ such that it introduces a rescaling factor $n$. We note that the integral multiplied by $1 / 2$ in the second equality is nothing but the QIM of the covering space, so we have

$$
G_{\lambda \lambda}^{\text {defect }}=n \cdot G_{\tilde{\lambda} \tilde{\lambda}}^{\mathrm{AdS}} .
$$

From this relation we conclude that the QIM of a conical defect is larger than the pure AdS case in general. The difference comes from the Virasoro algebra of the dual CFT of the conical defect. Specifically, $L_{0}$ has a fractionated spectrum (7), which results in the different central charges between two theories.

We make more remarks on this result now. As we claimed above, the theory dual to conical defect has a fractionated spectrum, which comes from the fact that the spectrum of $L_{0}$ is rescaled by $1 / n$ relative to $\tilde{L}_{0}$. That means the theory that is dual to the conical defect has smaller spectral gap so more internal d.o.f. than the one dual to the covering space. One can regard their relation as encapsulating $n$ d.o.f. of conical defect into a single d.o.f. 
of covering space, which leads to $\tilde{c}=c / n$ [13], because the central charge measures the d.o.f. of a quantum field. In other words, the dual quantum system of the conical defect is more complex than the covering one. On the other hand, the QIM also has the physical meaning of the fidelity susceptibility of the quantum system $\mathcal{F}(\lambda, \lambda+\delta \lambda)=$ $1-G_{\lambda \lambda} \delta \lambda^{2}+\mathcal{O}\left(\delta \lambda^{3}\right)$, which measures the distance between a perturbed ground state and its unperturbed ground state. So we can expect that as a marginal deformation with the same amplitude is added to the state dual to the conical defect and the covering space, respectively, more perturbative changes of the state will be observed for the conical defect compared to the covering, because of the greater number of d.o.f. and correlations. This results in less fidelity $\mathcal{F}^{\text {defect }}(\lambda, \lambda+\delta \lambda) \leq \mathcal{F}^{\mathrm{AdS}}(\tilde{\lambda}, \tilde{\lambda}+\delta \tilde{\lambda})$, and is consistent with (19) where $G_{\lambda \lambda}^{\text {defect }} \geq G_{\tilde{\lambda} \tilde{\lambda}}^{\text {AdS }}$.

\section{B. Bulk computation}

In this subsection we perform the holographic setup for an explicit check of our CFT calculation. We compute the QIM for an exactly marginal deformation in gravity side by using the holographic method. Here we just use a main result from [15] and more details can be found in Appendix A.

Let $Z_{2}$ be the partition function of a theory that only deforms the CFT for $\tau>0$, i.e.,

$$
\begin{aligned}
Z_{2}= & \int \mathcal{D} \varphi \exp \left(-\int_{-\infty}^{0} d \tau \int d^{d-1} x \mathcal{L}_{0}\right. \\
& \left.-\int_{0}^{\infty} d \tau \int d^{d-1} x\left(\mathcal{L}_{0}+\delta \tilde{\lambda} \tilde{\mathcal{O}}\right)\right) .
\end{aligned}
$$

One can write (A5) as

$$
\left\langle\Psi_{1} \mid \Psi_{0}\right\rangle=\frac{Z_{2}}{\left(Z_{1} Z_{0}\right)^{1 / 2}},
$$

where $Z_{0}$ is the partition function of the unperturbed theory and $Z_{1}$ is the partition function of the perturbed theory for $-\infty<\tau<\infty$ [see (A2) and (A4) in Appendix A].

The holographic dual conjecture allows us to calculate the partition functions of the CFT from the gravity side. Particularly in the large $N$ limit we can write the partition functions as $Z_{k}=e^{-I_{k}}$, where $I_{k}$ are the on-shell actions that correspond to the field theories with $k=0,1,2$, respectively. After a deformation, the on-shell action is perturbed by adding a contribution of a scalar field probing on the AdS space, $I_{k}=I_{\text {AdS }}+\delta I_{k}$, with

$$
\begin{aligned}
\delta I_{k} & =\frac{1}{2 \tilde{\kappa}^{2}} \int_{\mathcal{M}} d^{d+1} x \sqrt{g_{0}}\left(g_{0}^{\mu \nu} \partial_{\mu} \tilde{\Phi}_{k} \partial_{\nu} \tilde{\Phi}_{k}+m^{2} \tilde{\Phi}_{k}^{2}\right) \\
& =\frac{1}{2 \tilde{\kappa}^{2}} \int_{\partial \mathcal{M}} \sqrt{\gamma_{0}} n_{\mu} g_{0}^{\mu \nu} \tilde{\Phi}_{k} \partial_{\nu} \tilde{\Phi}_{k},
\end{aligned}
$$

where $g_{0}^{\mu \nu}$ is the unperturbed AdS metric. In the second line we have used the equation of motion and have written $\delta I_{k}$ as a boundary contribution by integrating by parts. $\tilde{\Phi}_{k}$ can be obtained by the boundary to bulk propagator in holography. Then one can write the overlap (21) as

$$
\begin{aligned}
\left\langle\Psi_{1} \mid \Psi_{0}\right\rangle & =\frac{Z_{2}}{\sqrt{Z_{1} Z_{0}}} \\
& =\exp \left(-I_{\mathrm{AdS}}-\delta I_{2}+\frac{1}{2}\left(I_{\mathrm{AdS}}+\delta I_{1}+I_{\mathrm{AdS}}\right)\right) \\
& =\exp \left(-\delta I_{2}+\frac{1}{2} \delta I_{1}\right)
\end{aligned}
$$

We now consider the QIM for the vacuum state of a CFT living on a cylinder. The operator $\tilde{\mathcal{O}}$ is dual to a probing scalar field in global AdS space whose metric is given by

$$
d s^{2}=\left(1+r^{2}\right) d \tau^{2}+\frac{d r^{2}}{1+r^{2}}+r^{2} d \Omega_{d-1}^{2},
$$

where we have set the AdS radius to 1 for simplicity.

We consider the marginal deformation, i.e., $\Delta=d$. Only $\delta I_{2}$ contributes to the QIM [15]. So we have only the boundary condition of the scalar field $\Phi_{2}$,

$$
\lim _{r \rightarrow \infty} \tilde{\Phi}_{2}(r, \tau)= \begin{cases}\delta \tilde{\lambda} & \text { if } \tau>0 \\ 0 & \text { if } \tau<0\end{cases}
$$

By adopting this boundary condition and solving the differential equation of $\tilde{\Phi}_{2}$, we can obtain the corresponding action contribution of probing scalar field $\delta I_{2}$ (23).

We turn to the QIM of conical defect. As shown above, for marginal perturbations, only $\delta I_{2}$ survives in (23). By definition, we have $G_{\lambda \lambda} \delta \lambda^{2}=\delta I_{2}^{\text {defect }}$. This implies that, to obtain the $G_{\lambda \lambda}$, we should find out the coupling constant $\delta \lambda$ and deformation $\delta I_{2}^{\text {defect }}$ for a conical defect. To proceed, we notice that there is a relation between scalar fields in the conical defect and the covering $\Phi(r=\infty, \tau, \theta)=$ $\tilde{\Phi}(r=\infty, \tau, \theta)$, and it can be understood as the following. The scalar wave functions in the conical defect or the covering space can be obtained by solving the KleinGordon equation $\left(\square-m^{2}\right) \Phi=0$ in the corresponding bulk geometry backgrounds. The solutions can be written as a usual ansatz [20,27]: $\Phi(r, t, \theta)=e^{i \omega t} Y_{l}(\theta) R(r)$. In covering space the angular dependent term $\tilde{Y}_{l}$ is solved as an eigenproblem and is given by

$$
\tilde{Y}_{l}(\theta)=e^{i l \theta}, \quad \tilde{Y}_{l}(\theta+2 a \pi)=\tilde{Y}_{l}(\theta), \quad l, a \in \mathbb{Z} .
$$

Similarly, the angular dependent term for conical defect can be written in the same form but with different periodicity [20], 

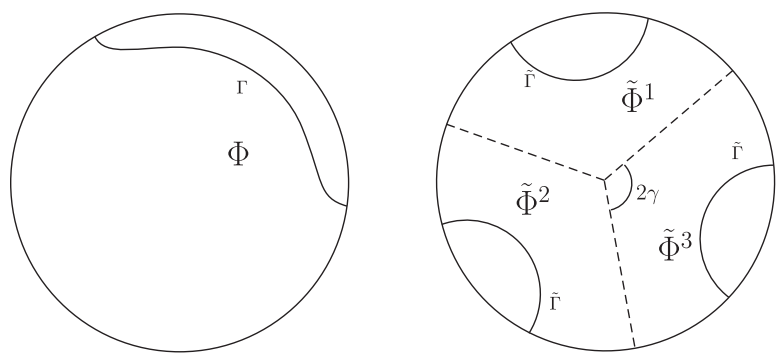

FIG. 1. (Left) Scalar field $\Phi$ on the conical defect geometry. $\Gamma$ is a minimal geodesic corresponding to a subregion of the system. It descends to a minimal geodesic $\tilde{\Gamma}$ on its covering space. (Right) The covering space of the conical defect. $\tilde{\Phi}^{i}$ are the scalar fields on it. $\Phi$ in the left is the $\mathbb{Z}_{n}$ symmetric $\tilde{\Phi}$ mode, which is a solution of equation in each conical defect copy. Let $\tilde{\Phi}^{i}$ denote the symmetrized modes of AdS in the $i$ th single copy.

$Y_{j}(\theta)=e^{i n j \theta}, \quad Y_{j}\left(\theta+\frac{2 b \pi}{n}\right)=Y_{j}(\theta), \quad j, b \in \mathbb{Z}$.

Therefore the $\Phi$ modes of the conical defect are a subset of the $\tilde{\Phi}$ modes with $l=n j$. In other words, the solution of the Klein-Gordon equation in the conical defect can be given by the $\mathbb{Z}_{n}$ symmetric $\tilde{\Phi}$ modes and restricting $\tilde{\Phi}$ to a single $\mathbb{Z}_{n}$ image, as shown in Fig. 1. Hence near the boundary we have the same coupling constants in the two theories, i.e., $\delta \lambda=\delta \tilde{\lambda}$. In the rest of this paper we only use $\delta \lambda$ to denote the coupling constant, and do not differentiate them from now on.

Next let us turn to the calculation of $\delta I_{2}^{\text {defect }}$. It is clear that to obtain the $\delta I_{2}^{\text {defect }}$, we simply need to sum over all contributions of the scalar fields probing the unperturbed background of all images in covering space,

$$
\begin{aligned}
\delta I_{2}^{\text {defect }}= & \frac{1}{2 \kappa^{2}} \sum_{k=0}^{n-1} \int_{\partial \mathcal{M}_{k}} \sqrt{\gamma_{0}} n_{\mu} g_{0}^{\mu \nu} \\
& \times\left.\Phi_{2}\left(\theta+\frac{2 \pi k}{n}\right) \partial_{\nu} \Phi_{2}\left(\theta+\frac{2 \pi k}{n}\right)\right|_{r=\infty} \\
= & \left.\frac{n}{2 \tilde{\kappa}^{2}} \int_{\partial \tilde{\mathcal{M}}} \sqrt{\gamma_{0}} n_{\mu} g_{0}^{\mu \nu} \tilde{\Phi}_{2} \partial_{\nu} \tilde{\Phi}_{2}\right|_{r=\infty},
\end{aligned}
$$

where $\partial \mathcal{M}_{k}$ and $\partial \tilde{\mathcal{M}}$ are the boundaries of the conical defect and the covering space, respectively. In the second line we have used the identity (17) once again to change the integral from conical defect $\partial \mathcal{M}$ to covering space $\partial \tilde{\mathcal{M}}$. We also change the gravitational constant by using $\tilde{\kappa}^{2}=n \kappa^{2}$. All these observations result in

$$
\delta I_{2}^{\text {defect }}=n \cdot \delta I_{2}^{\mathrm{AdS}},
$$

where $\delta I_{2}^{\mathrm{AdS}}$ is the perturbation of the covering space, which is just the pure AdS space. Recalling $G_{\lambda \lambda} \delta \lambda^{2}=\delta I_{2}$, we immediately get $G_{\lambda \lambda}^{\text {defect }}=n \cdot G_{\lambda \lambda}^{\text {AdS }}$, as expected.
By introducing a cutoff $\epsilon$, we get the following expression for the QIM of the conical defect (A20),

$$
G_{\lambda \lambda}^{\text {defect }}=\frac{n L^{d-1} \Gamma\left(\frac{1+d}{2}\right)}{2 \tilde{\kappa}^{2} \sqrt{\pi} \Gamma(d / 2)} \cdot \frac{\operatorname{Vol}\left(\mathbb{S}^{d-1}\right)}{(d-1) \epsilon^{d-1}}+\cdots,
$$

which has a cutoff independent universal constant.

\section{Comparing with the QIM/volume correspondence}

In [8] the authors argued that $G_{\lambda \lambda}$ of a $(d+1)$-dimensional CFT deformed by a marginal perturbation can be holographically estimated by

$$
G_{\lambda \lambda}=n_{d} \cdot \frac{\operatorname{Vol}\left(\Sigma_{\max }\right)}{L^{d}}
$$

where $n_{d}$ is a constant and $L$ is the $\operatorname{AdS}$ radius. $\operatorname{Vol}\left(\Sigma_{\max }\right)$ is the maximal volume of a $d$-dimensional spacelike surface in the bulk geometry, which is generally infinite and should be regularized with a cutoff $\epsilon$. Next we utilize our result to discuss this holographic conjecture. We find that the relation (31) is preserved. However, the coefficient $n_{d}$ in conical defect is different from the one in covering space.

In the global $\mathrm{AdS}_{d+1}$ case whose metric is given by (24), the QIM can be computed from the volume of a time slice. But we now prefer to use the coordinate (5) and we introduce the cutoff $\epsilon^{\prime}=n \epsilon$. When $n=1$ it is the pure AdS (we have set the AdS radius to 1),

$G_{\lambda \lambda}^{\mathrm{AdS}}=n_{d}^{\mathrm{AdS}} V_{d-1}^{\prime} \int_{0}^{r_{\infty}^{\prime}} \frac{r^{\prime d-1}}{\sqrt{r^{\prime 2}+1}} d r^{\prime}=\frac{n_{d}^{\mathrm{AdS}} V_{d-1}^{\prime}}{(d-1) \epsilon^{d-1}}+\cdots$,

where $V_{d-1}^{\prime}=\operatorname{Vol}\left(\mathbb{S}^{d-1}\right)$ and $\epsilon=\epsilon^{\prime}$ while $n_{d}^{\text {AdS }}$ is given by (A21) with $L=1$. Particularly in $d=2$, we have

$G_{\lambda \lambda}^{\mathrm{AdS}}(d=2)=n_{2}^{\mathrm{AdS}} V_{1}^{\prime} \cdot\left(\frac{1}{\epsilon}-1+\frac{\epsilon}{2}-\frac{\epsilon^{3}}{8}+\cdots\right)$,

which has a cutoff independent term.

We turn to estimate the QIM of conical defect in $d=2$ by using the coordinate (5). We calculate the volume of the time slice for a conical defect geometry and we obtain

$$
\begin{aligned}
G_{\lambda \lambda}^{\text {defect }} & =n_{2}^{\text {defect }} V_{1} \int_{0}^{r_{\infty}^{\prime}} \frac{r^{\prime}}{\sqrt{r^{\prime 2}+\frac{1}{n^{2}}}} d r^{\prime} \\
& =n_{2}^{\text {defect }} V_{1} \cdot\left(\frac{1}{\epsilon^{\prime}}-\frac{1}{n}+\frac{2 \epsilon^{\prime}}{2 n^{2}}-\frac{\epsilon^{\prime 3}}{8 n^{4}}+\cdots\right),
\end{aligned}
$$

where $n_{2}^{\text {defect }}$ is a coefficient in conical defect geometry. Replacing $\epsilon^{\prime}$ by $n \epsilon$ and comparing with our result (19), we have $n_{2}^{\text {defect }}=n^{2} \cdot n_{2}^{\text {AdS }}$. 
We observe that on the premise of generality of the argument (31), when we make a $\mathbb{Z}_{n}$ quotient of the original geometry, the coefficient $n_{2}$ should be rescaling by $n^{2}$. Generally speaking, in $(d+1)$ dimensions we have

$$
n_{d}^{\text {defect }}=n^{2} \cdot n_{d}^{\text {covering }}
$$

One more example comes from a geon black hole. It was shown in [28] how to construct a geon black hole. The $\mathbb{R P}^{2}$ geon can be constructed by taking a $\mathbb{Z}_{2}$ quotient of the action of a BTZ black hole. The BTZ black hole metric is given by $[29,30]$

$d s^{2}=-\left(\frac{r^{2}-r_{+}^{2}}{L^{2}}\right) d t^{2}+\left(\frac{L^{2}}{r^{2}-r_{+}^{2}}\right) d r^{2}+r^{2} d \theta^{2}$

The map $\tilde{J}$ of the $\mathbb{Z}_{2}$ quotient maps a point at $(t, \theta)$ in an asymptotical region to the one at $(-t, \theta+\pi)$ in another asymptotical region. The boundary manifold is a Klein bottle that is nonorientable. The $(d+1)$-dimensional AdS Schwarzschild planar black hole is analogous to the BTZ case. As a $\mathbb{Z}_{2}$ quotient of BTZ and AdS Schwarzschild planar black hole, our result implies

$$
n_{2}^{\mathrm{GEON}}=4 \cdot n_{2}^{\mathrm{BTZ}}, \quad n_{d}^{\mathrm{SAdSgeon}}=4 \cdot n_{d}^{\mathrm{SAdS}},
$$

which agree with the result in [28]. We can see this result is not related to the dimension $d$ because it comes from the quotient on the original manifold. The correlation function in geon dual theory is calculated by summing over two image contributions from the result of the quotient in BTZ $[31,32]$, i.e.,

$$
\left\langle\mathcal{O}(x) \mathcal{O}\left(x^{\prime}\right)\right\rangle_{\text {geon }}=\left\langle\mathcal{O}(x) \mathcal{O}\left(x^{\prime}\right)\right\rangle_{\mathrm{BTZ}}+\left\langle\mathcal{O}(x) \mathcal{O}\left(\tilde{J} x^{\prime}\right)\right\rangle_{\mathrm{BTZ}}
$$

where the first contribution comes from the BTZ in the same boundary and the second one is the two-point function of points in different boundary (as shown in Fig. 2). In [28] they also found that the second contribution to the QIM is same as the first one. So the contribution is double that of the original black hole, i.e., $G_{\lambda \lambda}^{\text {geon }}=2 G_{\lambda \lambda}^{\mathrm{BTZ}}$. On the other hand, the identification $(t, \theta) \sim(-t, \theta+\pi)$ shows a reflection around the vertical axis in the Penrose diagram of the BTZ black hole, as shown in Fig. 2. So the volume $\mathrm{Vol}^{\text {geon }}(\Sigma)$ is reduced to one half of the BTZ volume, i.e., $\mathrm{Vol}^{\text {geon }}(\Sigma)=\mathrm{Vol}^{\mathrm{BTZ}}(\Sigma) / 2$. Combining with (31), it gives a factor 4 on the coefficient (38).

We can also apply our method of holographic computation to the $\mathbb{R P}_{2}$ geon, which is absent in [28]; more details can be found in Appendix C. The result is consistent with the CFT computation.

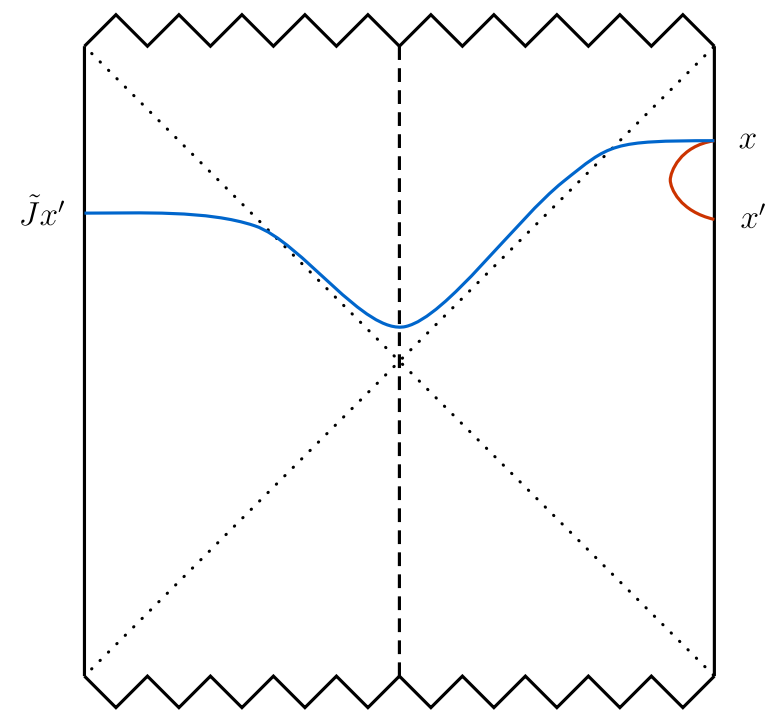

FIG. 2. The Penrose diagram of a BTZ black hole. The geon black hole can be obtained by a quotient of the BTZ, which is the vertical axis in the diagram. The two-point function includes two distinct contributions: one from correlations between two operators on the same boundary (red), the other from correlations between two operators on different boundaries (blue).

\section{MASSLESS BTZ BLACK HOLE}

In this section we consider a limit case $(n \rightarrow \infty)$ of the conical defect, which is the massless BTZ black hole. One can regard the conical defect as a spacetime which has a stationary particle at $r=0$. When the mass contribution of this particle approaches a critical value it becomes a black hole geometry with black hole mass $M=0$. The holographic duality of the massless BTZ black hole has been studied for many years $[16,17,33]$. However, in the present paper we only consider its QIM in the dual gravity side and give a rough picture without any field theoretical detail.

To proceed, let us start with the ordinary nonrotating BTZ black hole (37). The QIM of this black hole has been discussed in $[8,9,15,28]$ and explicitly it is given by

$G_{\lambda \lambda}^{\mathrm{BTZ}}=\frac{2}{\pi \tilde{\kappa}^{2}}\left[\frac{\pi V_{1}}{8 \epsilon_{\mathrm{BTZ}}}-\frac{\pi V_{1}}{2 \beta}+\frac{2 \pi^{2} V_{1}}{\beta^{2}} \cdot \tau \cot \left(\frac{4 \pi \tau}{\beta}\right)\right]$,

where we have set AdS radius to 1 and $V_{1}=2 \pi$. $\epsilon_{\mathrm{BTZ}}$ stands for the cutoff for the nonrotating BTZ. In order to match the two-point function on the CFT with the bulk side we multiply a constant $2 / \pi \tilde{\kappa}^{2}$ [15].

Then we notice that the massless BTZ black hole (MBTZ) can be obtained by taking a limit $M=J=0$ from the nonrotating BTZ black hole (37). Explicitly, the metric of MBTZ geometry is of the following form:

$$
d s^{2}=-\frac{r^{2}}{L^{2}} d t^{2}+\frac{L^{2}}{r^{2}} d r^{2}+r^{2} d \theta^{2}
$$


Above the metric shows that the MBTZ has an interesting feature: its horizon coincides with the singularity, i.e., $r_{+}=0$. This feature may lead to many nontrivial properties: there is no Hawking radiation of this black hole, i.e., $T_{H}=0$; the free energy (energy) and heat capacity (entropy) are also 0: $F_{\mathrm{MBTZ}}=-E_{\mathrm{MBTZ}}=0, C_{\mathrm{MBTZ}}=$ $S_{\mathrm{MBTZ}}=0$. Based on these properties, one naively thinks that the QIM can be obtained by taking the limit $\beta \rightarrow \infty$ in (40),

$$
G_{\lambda \lambda}^{\mathrm{BTZ}}(\beta \rightarrow \infty)=\frac{1}{4 \kappa^{2}} \cdot \frac{V_{1}}{\epsilon_{\mathrm{BTZ}}^{\prime}},
$$

where we have used the cutoff $\epsilon_{\mathrm{BTZ}}^{\prime}=n \epsilon_{\mathrm{BTZ}}$. So this remains a cutoff dependent quantity.

On the other hand, one can also obtain the MBTZ geometry by taking the $n \rightarrow \infty$ limit of the conical defect metric (5). So the results in this paper also hold for the MBTZ black hole. We take the limit $n \rightarrow \infty$ in the QIM of the conical defect (34), and the $\mathcal{O}\left(\epsilon^{\prime k}\right)(k \geq 1)$ terms are suppressed by large $n$ and small $\epsilon_{\mathrm{BTZ}}^{\prime}$; then it yields

$G_{\lambda \lambda}^{\text {defect }}(n \rightarrow \infty)=n_{2}^{\text {defect }}\left(\frac{V_{1}}{\epsilon_{\mathrm{BTZ}}^{\prime}}-\frac{V_{1}}{n}\right)=\frac{n^{2}}{4 \kappa^{2}}\left(\frac{V_{1}}{\epsilon_{\mathrm{BTZ}}^{\prime}}-\frac{V_{1}}{n}\right)$,

where we have used $n_{2}^{\text {defect }}=n^{2} \cdot n_{2}^{\text {AdS }}$ and $n_{2}^{\text {AdS }}=1 / 4 \kappa^{2}$ from (A21). Equations (42) and (43) indicate that in this limit the result does not exactly coincide with $G_{\lambda \lambda}^{\mathrm{BTZ}}(\beta \rightarrow \infty)$. Their cutoff dependent term has a similar behavior but different coefficient: $n_{2}^{\text {defect }}=n^{2} \cdot n_{2}^{\text {AdS }}$. In addition, $G_{\lambda \lambda}^{\text {defect }}(n \rightarrow \infty)$ has a cutoff independent universal term.

One natural question is the following: which one is the correct QIM of the MBTZ black hole. We argue that the information metric of the MBTZ is inclined to be obtained by taking $n \rightarrow \infty$ in the conical defect. The reason is the following.

First, as we have explained above, the conical defect geometry can be constructed by exciting a stationary point particle at $r=0$. This particle in the interior also contributes to the whole spacetime. One can obtain the whole contribution by integrating the spacetime stress tensor over $\theta$ and give the global contribution [18,34],

$$
M=-\frac{1}{8 G}+\frac{\gamma}{8 \pi G} .
$$

The first term in the rhs of (44) corresponds to the pure $\mathrm{AdS}_{3}$ contribution and the second term is the particle contribution, with $0 \leq \gamma \leq \pi$. Physically, the range $0<\gamma<\pi$ corresponds to the total mass $-1 / 8 G<M<0$. When $\gamma=0$ this is the pure AdS case. The range $M \geq 0$ corresponds to the spectrum of BTZ black holes (including MBTZ black holes). In conical defect geometry the angular coordinate range is $\theta \in[0,2 \pi A]$. We parametrize the conical defect as $A=1-4 G \mu$, where $\mu$ is the mass of the particle [12]. This particle creates a singularity at $r=0$ that results in the conical defect metric with the deficit angle (Fig. 1)

$$
\gamma=\pi(1-A)=4 \pi G \mu .
$$

Note that the particle mass $\mu$ differs from the ADM mass contribution (44) and they are related by [35]

$$
\mu=\frac{1}{4 G}(1-\sqrt{-8 G M}) .
$$

The subalgebra of the conical defect geometry only has Virasoro form when $A=1 / n$ [16], which leads to an $\mathrm{AdS}_{3} / \mathbb{Z}_{n}$ as considered in this paper. Comparing (45) and (46) we have

$$
M=-1 / 8 G n^{2}
$$

Clearly it becomes AdS as $n=1$ and a MBTZ black hole as $n=\infty$, as expected. This results in $n_{2}^{\text {defect }} / n_{2}^{\mathrm{AdS}}=$ $-1 / 8 G M$, where $-1 \leq M \leq 0$. So we find the coefficient of conical defect diverges when $M \rightarrow 0^{-}$(Fig. 3).

One more evidence is that the nonrotating BTZ and MBTZ are in different phases [35-38]. The authors in [37] provided some arguments that there is a second-order phase transition between the MBTZ and nonrotating BTZ because the energy and heat capacity are continuous. Here we can also see this phase transition by calculating the behavior of entropy in the bulk. To proceed, let us first focus on the free energy. The free energy of BTZ and conical defect was discussed in [39] and it can be obtained by calculating the on-shell action. To do this, first we need to regularize the divergence so it was suggested to consider the free energy of BTZ relative to $\mathrm{AdS}_{3}$ [39],

$-\log Z=I(\mathrm{BTZ})-I(\mathrm{AdS})=\frac{1}{4 \pi G}\left(\pi \beta-\pi^{2} r_{+}\right)$,

where $\beta$ is the inverse temperature of BTZ. The entropy of the BTZ black hole is then given by

$$
S_{\mathrm{BTZ}}=\left(1-\beta \frac{\partial}{\partial \beta}\right) \log Z_{\mathrm{BTZ}}=\frac{\pi r_{+}}{4 G} .
$$

To discuss the MBTZ black hole, we can choose the mass $M$ as an order parameter in the system. The entropy of the MBTZ black hole is then obtained by taking the limit $\beta \rightarrow \infty($ or $M \rightarrow 0)$,

$$
S_{\mathrm{BTZ}}(\beta \rightarrow \infty)=0,
$$

and the derivative with respect to $M$ is given by 

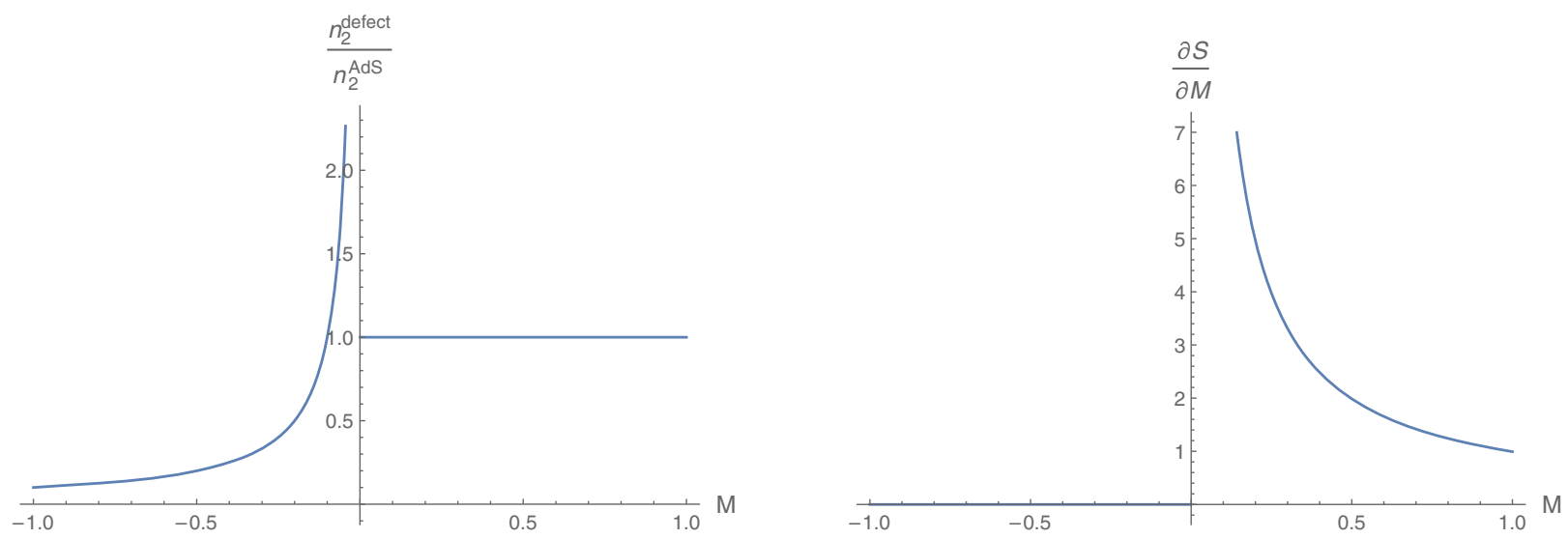

FIG. 3. We regard $M$ as an order parameter. (Left) The ratio of the coefficient for the conical defect to the one for the AdS. There is a divergence when $M \rightarrow 0^{-}$. (Right) First derivative of $S$ with respect to $M$. There is a divergence when $M \rightarrow 0^{+}$. This indicates a secondorder phase transition between the BTZ and MBTZ black hole. We set $8 G=10$.

$$
\left.\frac{\partial S_{\mathrm{BTZ}}}{\partial M}(\beta \rightarrow \infty) \sim \frac{1}{\sqrt{M}}\right|_{M \rightarrow 0} \rightarrow \infty
$$

We see that the derivative $\frac{\partial S_{\mathrm{BTZ}}}{\partial M}$ becomes infinite when the black hole tends to $M=0$.

On the other hand, the free energy of conical defect can be obtained very similarly; explicitly it is [39]

$-\log Z=I\left(X_{\chi}\right)-I(\mathrm{MBTZ})=-\frac{\beta}{8 G} \chi, \quad(0 \leq \chi \leq 1)$,

where $X_{\chi}$ denotes the manifold of the conical defect and $\chi$ is related to deficit angle through $\gamma=\pi(1-\sqrt{\chi})$. From (46) we have $n^{2}=1 / \chi$. In other words, the limit $n \rightarrow \infty$ implies $\chi \rightarrow 0$. We compute the free energy of conical defect relative to MBTZ (which is also $X_{0}$ ) rather than $\mathrm{AdS}_{3}$ because of the result matching with the partition function in dual CFT [39]. The free energy is proportional to $\beta$ so it has vanishing entropy for conical defect, $S_{X_{\chi}}=0$. The corresponding entropy and its derivative for $\chi \rightarrow 0$ (i.e., $M \rightarrow 0$ or $n \rightarrow \infty$ ) are

$$
\begin{aligned}
S_{X_{\chi}}(\chi \rightarrow 0) & =0, \\
\frac{\partial S_{X_{\chi}}}{\partial M}(\chi \rightarrow 0) & =0 .
\end{aligned}
$$

It is obvious that from MBTZ to massive BTZ there is a second order phase transition at $M=0$. As a contrast, there is nothing noncontinuous from conical defect to MBTZ (Fig. 3).

As a last point, if we consider the quantum effect in the BTZ black hole, Lifschytz and Ortiz argued in [24] that there is quantum instability of the MBTZ and concluded that the end of Hawking evaporation is not the MBTZ black hole but the AdS spacetime. So when the evaporation finishes $(M=0)$ we may not simply relate to MBTZ black hole in the bulk.

Based on all these observations, we conclude that one cannot simply make $\beta \rightarrow \infty$ in nonrotating BTZ black hole (40) to get properties for the MBTZ black hole. Instead, it is more safe to obtain them from the conical defect by taking $n \rightarrow \infty$.

\section{CONCLUSION AND DISCUSSION}

In this paper we have shown how to compute the QIM of the conical defect dual theory not only in the CFT side but also in the holographic point of view. In CFT, the correlation function in conical defect dual theory can be constructed by summing over nontrivial correlations between a different copy of images, which are embedded in a larger space. The larger space is the covering space of the conical defect space, which is pure $\mathrm{AdS}_{3}$. There are some nontrivial relations between the conical defect and its covering. One of the most important is that the Virasoro algebra of the conical defect dual theory is a subalgebra of that of its covering space. This leads to the fractionated spectrum and rescaled central charge $c=n \tilde{c}$. When we compute the QIM of the conical defect dual theory, this nontrivial property results in a rescaled information metric compared to the covering space: $G_{\lambda \lambda}^{\text {defect }}=n \cdot G_{\lambda \lambda}^{\text {AdS }}$. To verify our result, we perform a holographic computation, which is based on the method of [15]. Specifically, we sum over all scalar field deformations of the action $\delta I$. It turns out that for marginal perturbations, only $\delta I_{2}$ survives, and it can recover the result $G_{\lambda \lambda}^{\text {defect }}=n \cdot G_{\lambda \lambda}^{\text {AdS }}$.

We also consider the holographic description of the QIM, which is the maximal volume of a $d$-dimensional spacelike surface in the bulk geometry (31). After comparing with our result, we find that the dual relation for the conical defect is different from its covering by a coefficient, i.e., $n_{2}^{\text {defect }}=n^{2} \cdot n_{2}^{\text {AdS }}$. We argue that the difference comes from the $\mathbb{Z}_{n}$ quotient of the original space. This is further 
verified by the result in [28], where this coefficient for the geon black hole constructed by the $\mathbb{Z}_{2}$ quotient of the original black hole is different from its covering by a factor 4 , that is, $n_{2}^{\text {geon }}=4 \cdot n_{2}^{\mathrm{BH}}$. This factor 4 comes from the fact that the QIM of a geon black hole is double that of the original black hole and the volume of the geon black hole is half of that of the original one. We have a similar explanation for our conical defect result. In conical defect geometry, one copy of the conical defect is obtained by an identification $\theta \sim \theta+2 \pi / n$ of the $\mathrm{AdS}_{3}$ space. So the volume of the spacelike surface of the conical defect is $1 / n$ of that of the $\mathrm{AdS}_{3}$ space. Meanwhile, we have showed that the QIMs of the conical defect and its covering are related by $G_{\lambda \lambda}^{\text {defect }}=n \cdot G_{\lambda \lambda}^{\text {AdS }}$. So similar to the geon example, this gives a factor $n^{2}$ between the coefficient in two theories.

As to the MBTZ black hole, we find that the MBTZ black hole and the massive BTZ are not in the same phase; instead, MBTZ is in the same phase as the conical defect. This nontrivial property indicates that one cannot simply make $\beta \rightarrow \infty$ in nonrotating BTZ black hole (40) to get some properties of the MBTZ black hole. The right way is to obtain them from the conical defect by taking $n \rightarrow \infty$.

\section{ACKNOWLEDGMENTS}

This work was supported in part by the National Natural Science Foundation of China under Grants No. 11465012 and No. 11665016, and the 555 talent project of Jiangxi Province.

\section{APPENDIX A: QUANTUM INFORMATION METRIC IN HOLOGRAPHY}

In this Appendix we introduce the idea and some detailed computation for the QIM in $\mathrm{AdS}_{d+1}$, which mainly comes from [15]. We first review the CFT setup and then give a holographic perspective.
One can consider a $d$-dimensional CFT with Euclidean Lagrangian $\mathcal{L}_{0}$ and we add a deformation $\delta \mathcal{L}=\delta \lambda \mathcal{O}$ on it, where $\mathcal{O}$ is a primary operator with conformal dimension $\Delta$ and $\delta \lambda$ is a coupling constant of this deformation. The ground states of original theory and deformed theory are denoted by $\left|\Psi_{0}\right\rangle=|\Psi(\lambda)\rangle$ and $\left|\Psi_{1}\right\rangle=|\Psi(\lambda+\delta \lambda)\rangle$, respectively.

We use the path integral formalism to compute the overlap between the ground states of these two theories. First, for a generic state $|\tilde{\varphi}\rangle$ the overlap $\left\langle\tilde{\varphi} \mid \Psi_{0}\right\rangle$ can be written by

$$
\left\langle\tilde{\varphi} \mid \Psi_{0}\right\rangle=\frac{1}{\sqrt{Z_{0}}} \int_{\varphi(\tau=0)=\tilde{\varphi}} \mathcal{D} \varphi \exp \left(-\int_{-\infty}^{0} d \tau \int d^{d-1} x \mathcal{L}_{0}\right),
$$

where $Z_{0}$ is the partition function of the original theory,

$$
Z_{0}=\int \mathcal{D} \varphi \exp \left(-\int_{-\infty}^{\infty} d \tau \int d^{d-1} x \mathcal{L}_{0}\right)
$$

In the same way, the overlap $\left\langle\Psi_{1} \mid \tilde{\varphi}\right\rangle$ can also be written in a similar formalism,

$$
\begin{aligned}
\left\langle\Psi_{1} \mid \tilde{\varphi}\right\rangle= & \frac{1}{\sqrt{Z_{1}}} \int_{\varphi(\tau=0)=\tilde{\varphi}} \mathcal{D} \varphi \\
& \times \exp \left(-\int_{0}^{\infty} d \tau \int d^{d-1} x\left(\mathcal{L}_{0}+\delta \lambda \mathcal{O}\right)\right),
\end{aligned}
$$

where $Z_{1}$ is the partition function of the deformed theory,

$$
Z_{1}=\int \mathcal{D} \varphi \exp \left(-\int_{-\infty}^{\infty} d \tau \int d^{d-1} x\left(\mathcal{L}_{0}+\delta \lambda \mathcal{O}\right)\right) .
$$

Then the overlap $\left\langle\Psi_{1} \mid \Psi_{0}\right\rangle$ can be obtained as

$$
\begin{aligned}
\left\langle\Psi_{1} \mid \Psi_{0}\right\rangle & =\int \mathcal{D} \tilde{\varphi}\left\langle\Psi_{1} \mid \tilde{\varphi}\right\rangle\left\langle\tilde{\varphi} \mid \Psi_{0}\right\rangle \\
& =\frac{\int \mathcal{D} \varphi \exp \left(-\int_{-\infty}^{0} d \tau \int d^{d-1} x \mathcal{L}_{0}-\int_{0}^{\infty} d \tau \int d^{d-1} x\left(\mathcal{L}_{0}+\delta \lambda \mathcal{O}\right)\right)}{\left(Z_{0} Z_{1}\right)^{1 / 2}}
\end{aligned}
$$

We should be able to note that there is a discontinuity at $\tau=0$, which results in UV divergences. So we introduce a UV regularization $\epsilon$ at $\tau=0$ to make the path integral well defined. Then the $\left|\Psi_{1}\right\rangle$ is replaced by

$$
\left|\Psi_{1}(\epsilon)\right\rangle=\frac{e^{-\epsilon H_{0}}\left|\Psi_{1}\right\rangle}{\left(\left\langle\Psi_{1}\left|e^{-2 \epsilon H_{0}}\right| \Psi_{1}\right\rangle\right)^{1 / 2}}
$$

where $H_{0}$ is the Hamiltonian of the unperturbed theory. Now rewrite (A5) as the expectation value formalism in the unperturbed ground state,

$$
\left\langle\Psi_{1}(\epsilon) \mid \Psi_{0}\right\rangle=\frac{\left\langle\exp \left(-\int_{\epsilon}^{\infty} d \tau \int d^{d-1} x \delta \lambda \mathcal{O}(\tau, x)\right)\right\rangle}{\left\langle\exp \left(-\left(\int_{-\infty}^{-\epsilon}+\int_{\epsilon}^{\infty}\right) d \tau \int d^{d-1} x \delta \lambda \mathcal{O}(\tau, x)\right)\right\rangle^{1 / 2}} .
$$

We can expand the absolute value of the overlap (A7) for small $\delta \lambda$ to the second order; then we obtain

$$
\left|\left\langle\Psi_{1}(\epsilon) \mid \Psi_{0}\right\rangle\right|=1-G_{\lambda \lambda} \delta \lambda^{2}+\mathcal{O}\left(\delta \lambda^{3}\right) .
$$

The QIM is defined as minus the coefficient of the second order term $G_{\lambda \lambda}$. From (A7) we have 


$$
G_{\lambda \lambda}=\frac{1}{2} \int d^{d-1} x_{1} \int d^{d-1} x_{2} \int_{-\infty}^{-\epsilon} d \tau_{1} \int_{\epsilon}^{\infty} d \tau_{2}\left\langle\mathcal{O}\left(\tau_{1}, x_{1}\right) \mathcal{O}\left(\tau_{2}, x_{2}\right)\right\rangle
$$

where we have assumed the one-point function equals 0; i.e., $\langle\mathcal{O}\rangle=0$ for the operator in the original theory and the time reversal symmetry relation $\left\langle\mathcal{O}\left(-\tau_{1}, x_{1}\right) \mathcal{O}\left(-\tau_{2}, x_{2}\right)\right\rangle=\left\langle\mathcal{O}\left(\tau_{1}, x_{1}\right) \mathcal{O}\left(\tau_{2}, x_{2}\right)\right\rangle$ was used. We can also define a partition function $Z_{2}$ that is the theory only deformed for $\tau>0$,

$$
Z_{2}=\int \mathcal{D} \varphi \exp \left(-\int_{-\infty}^{0} d \tau \int d^{d-1} x \mathcal{L}_{0}-\int_{0}^{\infty} d \tau \int d^{d-1} x\left(\mathcal{L}_{0}+\delta \lambda \mathcal{O}\right)\right)
$$

Then the overlap (A5) can be written as

$$
\left\langle\Psi_{1} \mid \Psi_{0}\right\rangle=\frac{Z_{2}}{\left(Z_{1} Z_{0}\right)^{1 / 2}} .
$$

This is the general definition of QIM in the CFT side. We can also represent it in the gravity side.

The partition function of field theory can be obtained from the gravity partition function in the classical limit according to the AdS/CFT conjecture. In this classical regime, only saddle points contribute to the partition function; i.e., $Z_{k}=e^{-I_{k}}$, where $k=0,1,2$ and $I_{k}$ are the on-shell actions in the corresponding bulk.

Now let us turn to the bulk computation. From the holographic point of view, a probe scalar field in the bulk corresponds to a deformation by operator in the boundary theory. So in the bulk we should consider a scalar field perturbation to the unperturbed on-shell action; i.e., $I_{k}=I_{\mathrm{AdS}}+\delta I_{k}$. The perturbation is given by

$$
\begin{aligned}
\delta I_{k} & =\frac{1}{2 \kappa^{2}} \int d^{d+1} x \sqrt{g_{0}}\left(g_{0}^{\mu \nu} \partial_{\mu} \Phi_{k} \partial_{\nu} \Phi_{k}+m^{2} \Phi_{k}^{2}\right) \\
& =\frac{1}{2 \kappa^{2}} \int_{\partial \mathcal{M}} \sqrt{\gamma_{0}} n_{\mu} g_{0}^{\mu \nu} \Phi_{k} \partial_{\nu} \Phi_{k},
\end{aligned}
$$

where $\Phi_{1}$ and $\Phi_{2}$ satisfy different boundary conditions. Then we can represent the overlap as

$$
\begin{aligned}
\left\langle\Psi_{1} \mid \Psi_{0}\right\rangle & =\frac{Z_{2}}{\sqrt{Z_{1} Z_{0}}} \\
& =\exp \left(-I_{\mathrm{AdS}}-\delta I_{2}+\frac{1}{2}\left(I_{\mathrm{AdS}}+\delta I_{1}+I_{\mathrm{AdS}}\right)\right) \\
& =\exp \left(-\delta I_{2}+\frac{1}{2} \delta I_{1}\right)
\end{aligned}
$$

The scalar field that obeys the Laplace equation in the bulk has a leading term from the non-normalizable mode near the boundary. In other words, the boundary condition of this field is given by

$$
\lim _{r \rightarrow \infty} r^{d-\Delta} \Phi(r, \tau, \Omega)=\delta \lambda s_{k}(\tau),
$$

where

$$
s_{0}(\tau)=0, \quad s_{1}(\tau)=1, \quad s_{2}(\tau)= \begin{cases}1 & \text { if } \tau \geq 0 \\ 0 & \text { if } \tau \leq 0\end{cases}
$$

The subscript indicates that for different $Z_{k}$ we chose different boundary condition of the scalar field. We focus on the marginal deformation $\Delta=d$, which is only relevant to $\delta I_{2}$. Under the change of coordinates

$$
r=\frac{\cosh \rho}{\sinh T}, \quad \tau=\frac{1}{2} \log \frac{\cosh (T+\rho)}{\cosh (T-\rho)},
$$

with $T>0, \rho \in \mathbb{R}$, the global AdS metric changes to

$$
d s^{2}=d \rho^{2}+\left(\frac{\cosh \rho}{\sin T}\right)^{2}\left(d T^{2}+g_{\mathbb{S}^{d}-1}\right) .
$$

Together with the boundary condition, one can solve the equation of motion for $\Phi_{2}$; the solution is given by

$$
\Phi_{2}(\rho)=\frac{\delta \lambda \Gamma\left(\frac{1+d}{2}\right)}{\sqrt{\pi} \gamma(d / 2)} \int_{-\infty}^{\rho} \frac{1}{\cosh ^{d} x} d x .
$$

$\Phi_{2}$ only depends on $\rho$. By substituting $\Phi_{2}$ into the on-shell action, one obtains

$\delta I_{2}=\frac{L^{d-1} \delta \lambda^{2} \Gamma\left(\frac{1+d}{2}\right)}{2 \kappa^{2} \sqrt{\pi} \Gamma(d / 2)} \operatorname{Vol}\left(\mathbb{S}^{d-1}\right) \int_{\text {cutoff }}^{\infty} \frac{1}{\sinh ^{d} T} d T$,

where we have put a cutoff at $\sinh T=\epsilon$. After changing $r=(\sinh T)^{-1}$, the following QIM is obtained:

$G_{\lambda \lambda}^{\mathrm{AdS}}=\frac{L^{d-1} \Gamma\left(\frac{1+d}{2}\right)}{2 \kappa^{2} \sqrt{\pi} \Gamma(d / 2)} \operatorname{Vol}\left(\mathbb{S}^{d-1}\right) \int_{0}^{1 / \epsilon} \frac{r^{d-1}}{\sqrt{1+r^{2}}} d r$.

In [8] this prescription in the gravity side is simply by adding the defect brane action $S_{\text {brane }}=T \int_{\Sigma} \sqrt{g}$ to the Einstein-Hilbert action. When the deformation is infinitesimally small the brane tension is $T \simeq n_{d}^{\mathrm{AdS}} \delta \lambda^{2} / L^{d}$, where $n_{d}$ is fixed by 

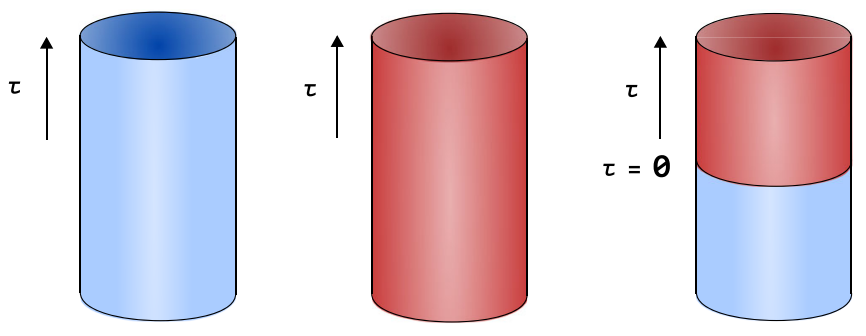

FIG. 4. A figure representation of configurations corresponding to $Z_{0}, Z_{1}$, and $Z_{2}$ on a cylinder. The blue color represents the undeformed theory and the red color represents the deformed theory induced by adding $\int \delta \tilde{\lambda} \tilde{\mathcal{O}}$ to the action.

$$
n_{d}^{\mathrm{AdS}}=\frac{L^{d-1} \Gamma\left(\frac{1+d}{2}\right)}{2 \kappa^{2} \sqrt{\pi} \Gamma(d / 2)},
$$

which is the coefficient in (A20). This is a universal constant in the $\mathrm{AdS}_{d+1}$ case.

So far we have derived the QIM in Poincaré $\operatorname{AdS}_{d+1}$. In this paper, we consider the global $\mathrm{AdS}_{3}$ case with its dual CFT living on a cylinder. The derivation of the QIM in this situation is similar to the Poincare case. The quantum information reads [26]

$$
\begin{aligned}
G_{\lambda \lambda}= & \frac{1}{2} \int d^{d-1} \Omega_{1} \sqrt{g_{S^{d-1}}} \int d^{d-1} \Omega_{2} \sqrt{g_{S^{d-1}}} \int_{-\infty}^{-\epsilon} d \tau_{1} \\
& \times \int_{\epsilon}^{\infty} d \tau_{2}\left\langle\mathcal{O}\left(\tau_{1}, \Omega_{1}\right) \mathcal{O}\left(\tau_{2}, \Omega_{2}\right)\right\rangle,
\end{aligned}
$$

where $Z_{0}$ is the partition function of the unperturbed theory, $Z_{1}$ is the partition function of the perturbed theory for $-\infty<\tau<\infty$, and $Z_{2}$ is the partition function of the theory that is only perturbed for $\tau>0$, as shown in Fig. $4 . \Omega$ is the compact spacelike dimension $\mathbb{S}^{d-1}$ and $\tau$ is the noncompact timelike dimension.

\section{APPENDIX B: NORMALIZATION CONSTANT OF THE TWO-POINT FUNCTION}

The derivation of the normalization constant of the twopoint function (15) was gotten by following [26]. The bulkto-boundary propagator in Poincaré coordinates is

$$
K\left(x ; x^{\prime}, z\right)=c_{\Delta} \frac{z^{\Delta}}{\left(z^{2}+\left|x-x^{\prime}\right|^{2}\right)^{\Delta}} .
$$

The coefficient $c_{\Delta}$ can be fixed by reaching to the boundary $z \rightarrow 0$,

$$
K\left(x ; x^{\prime}, z\right)=z^{d-\Delta} \delta^{d}\left(x-x^{\prime}\right) .
$$

Thus we have

$$
1=\int d^{d} x z^{\Delta-d} K\left(x ; x^{\prime}, z\right)=c_{\Delta} \int d^{d} x \frac{z^{2 \Delta-d}}{\left(z^{2}+\left|x-x^{\prime}\right|^{2}\right)^{\Delta}} .
$$

Algebraic calculation of the integral on the right-hand side yields

$$
c_{\Delta}=\left(\operatorname{Vol}\left(S^{d-1}\right) \frac{\Gamma\left(\frac{d}{2}\right) \Gamma\left(\Delta-\frac{d}{2}\right)}{2 \Gamma(\Delta)}\right)^{-1} .
$$

Using this bulk-to-boundary propagator the bulk scalar field can be obtained by

$$
\phi(x, z)=c_{\Delta} \int d^{d} x^{\prime} \frac{z^{\Delta} J\left(x^{\prime}\right)}{\left(z^{2}+\left|x-x^{\prime}\right|^{2}\right)^{\Delta}},
$$

where $J(x)$ is the boundary condition of $\phi(x, z)$. The normalization constant $\mathcal{N}_{\Delta, \kappa}$ defined in (15) has to be fixed from the holographic point of view: $\left\langle\exp \left(\int J \mathcal{O}\right)\right\rangle_{\mathrm{CFT}}=$ $\exp (-I)$, where $I$ is the on-shell action of the bulk theory,

$$
I=-\frac{1}{2 \kappa^{2}} \int_{z=\epsilon} d^{d} x\left(\sqrt{g} g^{z z} \phi \partial_{z} \phi\right) .
$$

Finally we have $\mathcal{N}_{\Delta, \kappa}=\ell^{d-1} c_{\Delta} d / \kappa^{2}$. The CFT on $\mathbb{R} \times S^{d-1}$ is related to the Poincaré case by a conformal map from plane to cylinder. So the two-point function in CFT on a cylinder has the same constant $\mathcal{N}_{\Delta, \kappa}$.

\section{APPENDIX C: THE BULK COMPUTATION OF QIM OF $\mathbb{R P}_{2}$ GEONS}

Similar to the conical defect case, we add a marginal perturbation in the ground state and see the change of the bulk action $\delta I_{2}$. In our argument this includes contributions from scalar fields in two images,

$$
\begin{aligned}
\delta I_{2}^{\text {geon }} \sim & \int \sqrt{\gamma_{0}} n_{\mu} g_{0}^{\mu \nu} \Phi_{2}(x) \partial_{\nu} \Phi_{2}(x) \\
& +\int \sqrt{\gamma_{0}} n_{\mu} g_{0}^{\mu \nu} \Phi_{2}(\tilde{J} x) \partial_{\nu} \Phi_{2}(\tilde{J} x) .
\end{aligned}
$$

To obtain $\delta I_{2}^{\text {geon }}$ we work in the unperturbed black hole metric, which can be written as

$$
d s^{2}=\frac{d Z^{2}}{\left(1-Z^{2}\right) Z^{2}}+\frac{1-Z^{2}}{Z^{2}} d \hat{\tau}^{2}+\frac{d \phi^{2}}{Z^{2}},
$$

where the following coordinate transformation has been performed:

$x=\sqrt{1-Z^{2}} \cos \hat{\tau} e^{\phi} \quad y=\sqrt{1-Z^{2}} \sin \hat{\tau} e^{\phi} \quad z=Z e^{\phi}$. 
To proceed, we first see the contribution from the first term, which is just the original BTZ. By introducing a cutoff at $Z=\epsilon e^{-\phi}$ we have [15]

$$
\delta I_{2}^{\mathrm{First}} \sim-\left.\int d \phi d \hat{\tau} \frac{e^{2 \phi}}{\epsilon} \Phi_{2} \partial_{z} \Phi_{2}\right|_{z=\epsilon},
$$

where we have set $r_{+}=1(\beta=2 \pi)$ for simplicity. The $\hat{\tau}$ integral runs over $\hat{\tau} \in[-\pi+\tau,-\tau]$. The scalar field $\Phi_{2}$ has the boundary condition similar to (25) with nonvanishing value in $\hat{\tau} \in[-\pi+\tau,-\tau]$. Since the integral is even in $\hat{\tau}$ we can only limit to $\hat{\tau} \in[-\pi / 2,-\tau]$ and then multiply a factor 2 in front of the integral. Then we shift the variable of integration $\hat{\tau}^{\prime}=\hat{\tau}-\epsilon$ and take the $\epsilon$ limit; then we obtain [15]

$$
\left.\partial_{z} \Phi\left(\hat{\tau}^{\prime}+\epsilon, \phi\right)\right|_{z=\epsilon}=\frac{\delta \lambda}{\pi} e^{-2 \phi} \mathcal{F}\left(\hat{\tau}^{\prime}\right) \epsilon,
$$

where

$$
\begin{aligned}
\mathcal{F}(\hat{\tau})= & \frac{\mathcal{F}_{1}(\hat{\tau})}{2(\cos (2 T)-\cos (2 \hat{\tau}))^{2}}, \\
\mathcal{F}_{1}(\hat{\tau})= & 2 \cos (2 \hat{\tau})[\sin (2 T)+\pi \cos (2 T)]+h_{1}(\hat{\tau}) \\
& +h_{1}(-\hat{\tau})-\sin (4 T)-2 \pi,
\end{aligned}
$$

and $h_{1}$ is a function of $\hat{\tau}$. Finally, by integrating the $\phi$ in $\delta I_{2}$ we have

$$
\begin{aligned}
\delta I_{2}^{\mathrm{First}} & \sim-\frac{\delta \lambda^{2} V_{1}}{\pi} \int_{-\pi / 2-\epsilon}^{-\tau-\epsilon} d \hat{\tau}^{\prime} \mathcal{F}\left(\hat{\tau}^{\prime}\right) \\
& =\frac{\delta \lambda^{2} V_{1}}{\pi}\left(\frac{\pi}{2 \epsilon}+\frac{-1+2 \tau \cot (2 \tau)}{2}\right) .
\end{aligned}
$$

Now we turn to consider the second contribution in (C1). After a changing of coordinates $Z=r_{+} / r, r_{+} \hat{\tau}=i t$, and $\phi=r_{+} \theta$, we get the Lorentzian metric (37) by setting $r_{+}=1$. So the second contribution comes from the quotient $(\hat{\tau}, \phi) \sim(-\hat{\tau}, \phi+\pi)$. We can simply replace $(\hat{\tau}, \phi)$ with $(-\hat{\tau}, \phi+\pi)$ in the integral (C4) and obtain

$$
\delta I_{2}^{\text {Second }} \sim-\frac{\delta \lambda^{2} V_{1}}{\pi} \int_{-\pi / 2-\epsilon}^{-\tau-\epsilon} d \hat{\tau}^{\prime} \mathcal{F}\left(-\hat{\tau}^{\prime}\right)
$$

We note that this integral is independent of $\phi$ and $\mathcal{F}(\hat{\tau})$ is even in $\hat{\tau}$, i.e., $\mathcal{F}(-\hat{\tau})=\mathcal{F}(\hat{\tau})$. So the second contribution is the same as the first one, i.e., $\delta I_{2}^{\text {Second }}=\delta I_{2}^{\text {First }}$. Finally the QIM of a geon black hole is double that of the original black hole, which agrees with the CFT computation.
[1] J. Maldacena, The large-N limit of superconformal field theories and supergravity, Adv. Theor. Math. Phys. 2, 231 (1998).

[2] E. Witten, Anti-de Sitter space and holography, Adv. Theor. Math. Phys. 2, 253 (1998).

[3] S. Ryu and T. Takayanagi, Holographic Derivation of Entanglement Entropy from AdS/CFT, Phys. Rev. Lett. 96, 181602 (2006).

[4] S. Ryu and T. Takayanagi, Aspects of holographic entanglement entropy, J. High Energy Phys. 08 (2006) 045.

[5] L. Susskind, Entanglement is not enough, Fortschr. Phys. 64, 49 (2016).

[6] L. Susskind, Computational complexity and black hole horizons, Fortschr. Phys. 64, 24 (2016); Addendum, Fortschr. Phys. 64, 44 (2016).

[7] D. Stanford and L. Susskind, Complexity and shock wave geometries, Phys. Rev. D 90, 126007 (2014).

[8] M. Miyaji, T. Numasawa, N. Shiba, T. Takayanagi, and K. Watanabe, Distance between Quantum States and GaugeGravity Duality, Phys. Rev. Lett. 115, 261602 (2015).

[9] D. Bak, Information metric and Euclidean Janus correspondence, Phys. Lett. B 756, 200 (2016).

[10] W. C. Gan and F. W. Shu, Holographic complexity: A tool to probe the property of reduced fidelity susceptibility, Phys. Rev. D 96, 026008 (2017).
[11] S. Deser, R. Jackiw, and G. 't Hooft, Three-dimensional Einstein gravity: Dynamics of flat space, Ann. Phys. (N.Y.) 152, 220 (1984).

[12] S. Deser and R. Jackiw, Three-dimensional cosmological gravity: Dynamics of constant curvature, Ann. Phys. (N.Y.) 153, 405 (1984).

[13] V. Balasubramanian, B. D. Chowdhury, B. Czech, and J. de Boer, Entwinement and the emergence of spacetime, J. High Energy Phys. 01 (2015) 048.

[14] V. Balasubramanian, A. Bernamonti, B. Craps, T. De Jonckheere, and F. Galli, Entwinement in discretely gauged theories, J. High Energy Phys. 12 (2016) 094.

[15] A. Trivella, Holographic computations of the quantum information metric, Classical Quantum Gravity 34, 105003 (2017).

[16] J. de Boer, M. M. Sheikh-Jabbari, and J. Simon, Near horizon limits of massless BTZ and their CFT duals, Classical Quantum Gravity 28, 175012 (2011).

[17] V. Balasubramanian, P. Kraus, and M. Shigemori, Massless black holes and black rings as effective geometries of the D1-D5 system, Classical Quantum Gravity 22, 4803 (2005).

[18] V. Balasubramanian and S. F. Ross, Holographic particle detection, Phys. Rev. D 61, 044007 (2000).

[19] J.D. Brown and M. Henneaux, Central charges in the canonical realization of asymptotic symmetries: An example 
from three-dimensional gravity, Commun. Math. Phys. 104, 207 (1986).

[20] I. Y. Aref'eva and M. A. Khramtsov, AdS/CFT prescription for angle-deficit space and winding geodesics, J. High Energy Phys. 04 (2016) 121.

[21] D. S. Ageev, I. Y. Aref'eva, and M. D. Tikhanovskaya, $(1+1)$-correlators and moving massive defects, Teor. Mat. Fiz. 188, 85 (2016) [Theor. Math. Phys. 188, 1038 (2016)].

[22] I. Y. Arefeva and A. A. Bagrov, Holographic dual of a conical defect, Teor. Mat. Fiz. 182, 3 (2015) [Theor. Math. Phys. 182, 1 (2015)].

[23] E. Keski-Vakkuri, Bulk and boundary dynamics in BTZ black holes, Phys. Rev. D 59, 104001 (1999).

[24] G. Lifschytz and M. Ortiz, Scalar field quantization on the $(2+1)$-dimensional black hole background, Phys. Rev. D 49, 1929 (1994).

[25] T. Banks, M. R. Douglas, G. T. Horowitz, and E. Martinec, AdS dynamics from conformal field theory, arXiv:hep-th/ 9808016.

[26] D. Bak and A. Trivella, Quantum information metric on $\mathbb{R} \times S^{d-1}$, J. High Energy Phys. 09 (2017) 086.

[27] J. C. Cresswell and A. W. Peet, Kinematic space for conical defects, J. High Energy Phys. 11 (2017) 155.

[28] M. Sinamuli and R. B. Mann, Geons and the quantum information metric, Phys. Rev. D 96, 026014 (2017).

[29] M. Banados, C. Teitelboim, and J. Zanelli, The Black Hole in Three-Dimensional Spacetime, Phys. Rev. Lett. 69, 1849 (1992).
[30] M. Banados, M. Henneaux, C. Teitelboim, and J. Zanelli, Geometry of the $(2+1)$ black hole, Phys. Rev. D 48, 1506 (1993); Erratum, Phys. Rev. D 88, 069902(E) (2013).

[31] J. Louko, D. Marolf, and S. F. Ross, Geodesic propagators and black hole holography, Phys. Rev. D 62, 044041 (2000).

[32] M. Guica and S. F. Ross, Behind the geon horizon, Classical Quantum Gravity 32, 055014 (2015).

[33] A. Strominger, Black hole entropy from near horizon microstates, J. High Energy Phys. 02 (1998) 009.

[34] V. Balasubramanian and P. Kraus, A stress tensor for anti-de Sitter gravity, Commun. Math. Phys. 208, 413 (1999).

[35] Y.S. Myung, No Hawking-Page phase transition in three dimensions, Phys. Lett. B 624, 297 (2005).

[36] Y.S. Myung, Phase transition between the BTZ black hole and AdS space, Phys. Lett. B 638, 515 (2006).

[37] R. G. Cai, Z. J. Lu, and Y.Z. Zhang, Critical behavior in $(2+1)$-dimensional black holes, Phys. Rev. D 55, 853 (1997).

[38] R. Abt, J. Erdmenger, H. Hinrichsen, C. M. MelbyThompson, R. Meyer, C. Northe, and I. A. Reyes, Topological complexity in AdS3/CFT2, Fortschr. Phys. 66, 1800034 (2018).

[39] J. R. David, G. Mandal, S. Vaidya, and S. R. Wadia, Point mass geometries, spectral flow and $\operatorname{AdS}(3)$-CFT(2) correspondence, Nucl. Phys. B564, 128 (2000). 